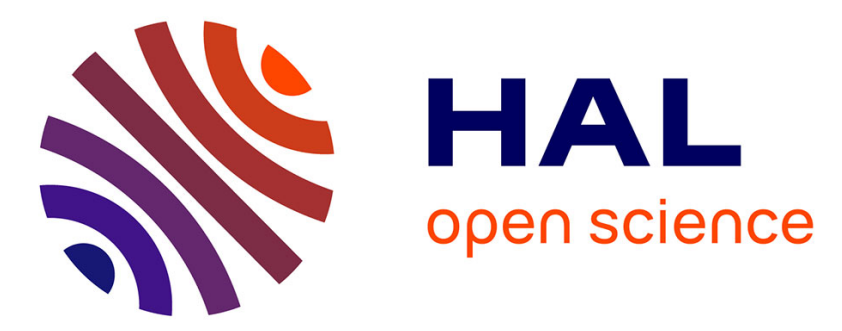

\title{
La mort des SDF à Paris: un révélateur social implacable
}

Daniel Terrolle

\section{- To cite this version:}

Daniel Terrolle. La mort des SDF à Paris : un révélateur social implacable. Etudes sur la mort. Revue de la Société de thanatologie, 2002, 122, pp.55-68. halshs-00067743

\section{HAL Id: halshs-00067743 \\ https://shs.hal.science/halshs-00067743}

Submitted on 11 May 2006

HAL is a multi-disciplinary open access archive for the deposit and dissemination of scientific research documents, whether they are published or not. The documents may come from teaching and research institutions in France or abroad, or from public or private research centers.
L'archive ouverte pluridisciplinaire HAL, est destinée au dépôt et à la diffusion de documents scientifiques de niveau recherche, publiés ou non, émanant des établissements d'enseignement et de recherche français ou étrangers, des laboratoires publics ou privés. 
Daniel Terrolle

« La mort sociale des SDF à Paris : Un révélateur social implacable »

Etudes sur la mort, 2002, nº122, pp.55-68

ISSN : 1157-0466

Revue de la société de thanatologie éditée par les Editions l'Esprit du Temps

\section{LA MORT DES SDF À PARIS : UN RÉVÉLATEUR SOCIAL IMPLACABLE}

«Toutes (les sociétés) mentent comme elles respirent, mais il faut bien respirer pour vivre. »

Pouillon J., (1977).

«Plus c'est la même chose, plus ça change. »

Nouvelle Revue de Psychanalyse, 15.

L'anthropologue qui s'attache à l'étude du vivant aurait tort d'ignorer ce que l'étude de la mort de ce dernier peut lui apporter dans la connaissance de son objet : de fait, la manière dont chaque société traite ses morts révèle de manière exemplaire, à travers ce rite de passage ultime ou son absence, des significations essentielles sur l'ordre social qui l'anime, au-delà de la précaution des représentations dont elle s'entoure.

L'étude de la mort apporte à la compréhension du « tissu social » l'ne lecture de son envers autant et sinon plus révélatrice que celle de son endroit. Elle rend compte de cette face cachée, de ce visage obscur qui, en se dévoilant ainsi offre une vérité d'autant plus redoutable qu'elle se donne à lire, dans la trutalité des faits de terrain, c'est-à-dire dépouillée de tous les artifices et des enluminures symboliques, tant des gestes que de la parole.

C'est à la découverte de cette face cachée, relative à la mort des SDF à Paris, que le lecteur est convié tout en l'avertissant que l'approche de celle-ci ne se fait qu'au prix de questionnements constants, tant ne va pas de soi, sur un sujet dominé par les représentations et, à la limite tout entier défenciu par la force de celles-ci, compte tenu des enjeux sociaux qui s'y jouent, l'irruption même de la question de leur mort. 


\section{UN CONSTAT SCIENTIFIQUE TROUBLANT}

À la suite de recherches menées depuis 1991 sur le mode de vie des SDF à Paris 1, et en 1994-1995, sur les équipements dissuasifs urbains conçus à leur encontre, la question de leur avenir a surgi au détour d'un double constat : d'une part, les responsables des associations caritatives attestaient tous régulièrement du volum: constant si ce n'est en sugmentation de cette population et d'autre part, les médecins qui la soignaiert témoignaient tous, comme en aparté, de sa faible espérance de vie. La réso'ution logique de ces assertions ne scmblait pas poser problème. En effet, on pouvait immédiatement conclure que si cette population se maintenait ou inême augmentait (en volume), malgré une espérance de vie courte, c'est qu'eile enregistrait un renouvellement constant au moins ég.ll sinon supérieur au nombre de ceux qui la quittaient. Or, il n'y a pas d'autres issues pour sortir de la vic à la rue que l'alternative qui est soit de se réinsérer, soit de mourir. La question se pose donc de manière simple et $\mathrm{s}$. résolution ne pcut passer que par l'examen successif de ces deux occurrences.

Un premier bilan des publications parues sur chacune d'entre elles met en évidence un déricit manifeste entre la pléthore des ouvrages relatifs à l'insertion (ou à la réinsertion) et l'absence totale de publications françaises sur la mort des SDF. Ce premier indice, troublant, motiva le chercheur à dépouiller ces sources pour l'amener à constater que l'abondante littérature produite à propos de l'insertion concerne essentiellement les dispositifs et financements mis en place à cette fin, tout comme les conseils et recommandations en tout genre à même de la réaliser. Or, pour contrebalancur le caractère théorique et virtuel de ces démonstratiuns, peu de faits patcn.s sont recensés et analysés quand seulement l'auteur pense à prouver ainsi la pertinence de ses analyses théoriques. Ainsi, des évidences toutes faites s'y affinnent plus qu'elles ne s'y démontrent, reposant plus sur une croyance dans le bien fondé des dispositifs que sur les résultats manifestes de leur prétendue performance.

Bien plus troublante encore est l'attitudk consensuelle de toute une sociologie du «social » qui, après s'être évertuće à mettre en évidence la «carrière » du SDF - c'est-à-dire ce qui amenait ces personnes à se retrouver à la rue - interrompt brutalement ses recherches et suspend sa curiosité scientifique quand ii s'agit d'analyser ce qu'elles deviennent et comment cette carrière s'achève. Si certains concluent alors à la nécessité de la réinsertion comme un vœu pieux, d'autres ${ }^{2}$, sans amener la moindre prcuve de terrain, laissent entendre que : «...S'il y a des entrées dans la carrière des sans donicile, il y a aussi des sorties ». Justement, on

1. Terrolie D., Dir., 1993, «Enances urbaines. Recherche en ethnologie urbaine », Rapport pour le PUCA, $230 \mathrm{p}$.

2. Darr on J., Firdion J. M., 1996, «Vivre dans la rue : la question SDF », S. Paugam Dir., L'exclusion l'átat des savoirs, Paris, Là Découverte, 583 p. aurait aimé savoir lesquelles... De la part d'auteurs dont on ne peut que partager la conclusion, a laquelle malhcureusement ils dérogent, lorsqu'ils écrivent : "Affirmer la nécessité de la connaissance et de l'évaluation permet de sortir du domaine de l'incantation et de l'idéologie qui trop souvent entoure les dispositifs d'aide aux $S D F \gg 3$.

Un dernier constat vient enfin parachever le trouble sur ce sujet : la France possède deux observatoires de la pauvreté chargés d'assurer une veille attentive à toutes les questions concernant les personnes les plus défavorisées. Or, la question de la mort des SDF n'a jamais fatit l'objet de la moindre interrogation tant dans les publications de l'Observatoire National de la Pauvreté et de l'Exclusion Sociale que dans celles de l'Observatoire du Samu Social de Paris. Plus curieusement encore, cette dernière institution réussit le tour de force d'éviter le surgissement de cette question dans les rapports épidémiologiques qu'elle produit régulièrement et dans lesquels elle cite même des travaux étrangers où des données relatives à ce fait sont analysées ! À ce stade, comment ne pas s'interroger sur le mutisme délibéré dont semblent faire preuve ces institıtions dont la mission revendiquée et les financements obtenus visent justement à rendre compte de tous les problèmes relatifs aux parsonnes les plus défavorisées? La mort de ces personnes serait-elle un problème annexe, surtout à la lumière de leur état épidémiologique? Si aucune logique scientifique ne peut justifier catte attitude délibérée, il faut chercher ailleurs les raisons qui fondent l'évitement systématique de cette question. En quoi la mort des SDF serait-elle une question dangereuse pour mobiliser à son sujet tant d'occultations systématiques ?

\section{L'IMAGINAIRE DE L'INSERTION}

L'état des lieux fait apparaître d'abord l'absence de toute statistique pertinente relative à l'insertion. Bertrand Bergier, l'un des rares sociologues à avoir travaillé sur cette question 4 atteste que : «Vraisemblablement, sur le plan statistique, Catherine et les autres représentent une quantité négligeable », ce n'est pas pour autant que leurs itinéraires doivent être négligés ». Il s'étonne, à juste titre, que la sociologie n'ait pas poussé ses analyses jusque-là 5 .

\section{Ibidem, p.385.}

4. Bergier B., 2000, Les Affranchis. Étiquetés «SDF, drogués, marginaux, inemployables... » Ils s'en sont sortis!, Paris, L'IJarmattan, $207 \mathrm{p}$.

5. "Quand bien même serions-nous dans une sociologie de l'exception, l'observation altentive d'un phénomène aberrant offre deux issues dignes d'intérêt : soit lui donner une place dans un modèle thérique existant, place qu'il n'a pu alors trouver faute de donner une place ce qui : fffine le modèle; soit montrer l'inadéquation de cette construction théorique et aboutir à une nouvelle modélisation », Ibidem, p. 6. 
l'our notre part, une rapide enquête de terrain menée auprès des responsables d'associations caritatives confirme cela: ces derniers, tout en reconnaissan que toute « sortie » de leur association ne s'assimile pas à une réinsertion réussie (la personne entrant souvent alors dans une autre association) n'en continuent pas moins de comptabiliser ces sorties comme autant de preuves de l'efficacité du système. Foussés dans leurs retranchements, ils finissent par avouer qu'en dehors des «pauvres salariés » obligés d'avoir recours aux dispositifs d'hébergemen et d'alin entation d'urgence (à sause de la faiblesse de leurs revenus issus de contrats précaires) on peut estimer à $1 \%$ les personnes qui, tombés à la rue, rebondissent très rapidement et se réinsèrent dans l'économie englobante, à 4 a $5 \%$ ccux qui, après quelques mois de vie à la rue, finissent, au terme d'échecs réitérés el de démarches, par s'insérer dans une économie protégée 6 . Quant aux autres, soit 94 à $95 \%$, si certains témoignent « ne pas savoir ce qu'ils deviennent», d'autres finissent par reconnaître qu'ils «disparaissent », qu'ils «meurent», compte tenu de la dureté des conditions de vie à la rue. Ces évaluations ne sont pas nouvelles : déjà le journaliste H. Prolongeau, en 1993 dressait le même constat en citant $X$. Emmanuelli : «La réinsertion est une illusion pour beaucoup de SDF, pour lesquels il n'y a rien à faire d'autre que les aider » et P. Declerck 7 .

Face à un tel constat qui peıdure plusieurs questions surgissent : pourquoi cette attitide à propos de la mort des SDF s'il en meurt plus qu'il ne s'en réinsère ? À quoi et à qui servent alors les financements publics conséquents versés par l'État et les collectivités locale s pour l'insertion?

\section{LA MORT DES SDF : L’ESCAMOTAGE DÉLIBÉRÉ D’UNE RÉALITÉ8.}

De l'alternative évoauée quant au devenir des SDF, il ne reste plus qu'à analyser le dernier terme, c'est-à-dire leur mort. Quatre années de recherche seront nécessaires à cela, pour déméler les fils multiples des faits, pour surmonter les réticences, pour patiemment repérer les «non-dits», pour comprendre les silences embarrassés, les réponses évasives et le sens des fins de non recevoir concernant aussi bien le questionnement de l'ethnologue que, parfois, l'ethnologue lui-ıême.

6. Cette économie protégée peut être le fait de structures caritatives ou du détournement de structures publiques comme les Centre.s d'Aide par le Travail, initialement destinés aux handicapés mentaux et qui accueillent maintenant de plus en plus d' "handicapés sociaux ".

7. Ce dernier en témoigne ouvertument dans Les naufragés, Plon, 2001, au chapitre « De la charité hystérique à la fonction asilaire ", p. 319 et suivantes.

8. Les élements essentiels de cutte enquête ont déjà été publiés in Terrolle D., 1999, Privés de deuil, Le Nouveau Mascaret, Bordeaux, CREAHI., n 55, pp. 26-32.
Pendant ce temps, les lois sur la bioéthique se mettront en place, modifian certains faits ; certaines structures changeront, dispersant les informateurs, cel nécessitant à chaque fois de réajuster l'enquête, de réactualiser les données.

Deux pistes complémentaires s'offrent à la compréhension du phénomène l'une est quantitative, statistique, alors que l'autre est qualitative, ethnographique.

\section{DES STATISTIQUES MUETTES}

La première tourne court très vite: déjà hypothéquée par une absence de définition statistique de son vivant, la personne à la rue reste indécelable dans les statistiques de mortalité. La démonstration peut s'arrêter là. Cependant elle serait incomplète dans ce qu'elle révèle des multiples dysfonctionnements techniques mais aussi politiques qui y président.

Basées sur le traitement des builetins de décès, les statistiques relatives à la mortalité de la population à la rue, sont tout d'abord biaisées par le classement des SDF par l' INSEE dans la vaste catégorie des inactifs (avec les retraités, les rentiers, les femmes au foyer et autres). À ce propos, divers responsables de cette institution finiront par admettre que si des difficultés techniques existent pour cerner au plus près cette population, « elles ne sont pas insurmontables si la volonté politique d'y arriver se manifeste $»$. Pourquoi celle-ci ne se manifeste-t-elle pas ? Pourquoi, comme le suggère un spécialiste de cette question, la volonté de suivre jusqu'à la mort une cohorte de personnes à la rue n'a-t-elle jamais été effectuée, alors que les SDF existent depuis plus de vingt ans en France?

À cette dilution statistique globale des vivants s'ajoute, constatée par l'INSERM qui traite plus particulièrement des causes de mortalité, l'absence systématiquc de données, des résultats d'autopsies, provenant de l'Institut médicolégal, par lequel passent tous les cadavres retrouvés à la rue, à Paris. Contactée, cette dernière institution, malgré une présentation argumentée de cette recherche, refusera obstinément de rencontrer le chercheur. Or, ce dernier apprendra, qu'audelà du désert statistique officiel sur la mortalité de ces personnes, les chiffres existent au Ministère de l'Intérieur (dont dépend l'IML). Pourquoi rıe sont-ils pas rendus publics? En quoi la mort des SDF relève-t-elle d'un quelconque secret?

\section{L'ENSEIGNEMENT DU TERRAIN}

L'ensemble de ces causes évoquées prend sens à la lumière de l'examen de la piste qualitative, c'est-à-dire lors de l'enquête de terrain. Son projet était de cerner d'abord les divers lieux de cette mort: deux champs s'imposaient, l'un à l'hôpital où la personne à la rue pouvait décéder suite à son hospitalisation, l'autre était les espaces publics où son cadavre était trouvé. 
Dans sa grande candeur, le chercheur pensait naïvement que la connexion informatique en réseau des diverses institutions hospitalières, sur Paris, serait à même de lui fournir des éléments pertinents et rapides sur ce sujet : il déchanta rapidement. D'une part, cette dernière n'était pas opérationnelle pour traiter de cela, d'autre part, en raison de logiques internes de fonctionnement parfois obscures (comme le fait qu'on ne décède pas en réanimation, même si c'est le cas...), la recherche des informations (et à plus forte ıaison des dossiers de personnes décédées à l'hôpital) relève du parcours du combattant, rendu encore plus compliqué par l'éthique médicale (qui fait relever l'approche de ces questions de la seule compétence d'un médecin). Or, curieusement, aucun médecin, sur Paris, bien que conscient de la faible espérance de vie de ces personnes, ne semble se précipiter pour étudier leur mortalité 9 .

L'enquête de terrain relative au devenir du cadavre trouvé dans les espaces publics a permis d'établir les étapes suivantes :

- Dès sa découverte et l'appel fait à la police ou aux Pompiers de Paris, ces services sollicitent un médecin de l'IML pour constater le décès et acheminer le corps jusqu'à cette institution pour déterminer les causes de la mort.

- C'est à l'IML que, d'une part, l'identité du défunt sera recherchée, et que d'autre part, un premier examen du corps, articulé au constat dressé lors des circonstances de sa découverte permettra d'établir s'il s'agit d'une mort «naturelle » ou «suspecte». Dans ce dernier cas, le parquet est saisi, ordonne une autopsie qui, si elle confirme les présnmptions du premier examen, permettra de lancer une cnquête policière. Concernant l'identification du corps, il n'est pas rare que celui-ci soit retrouvé dépourvu de papiers d'identité : la brigade d'identification judiciaire est alors chargée d'établir cette dernière. Jusqu'à un passé récent, une photo «post mortem » présentée à la secrétaire du CHAPSA permettait d'avoir des renseignements, mais cette dernière ayant changé de service et d'établissement, c'est au personnel permanent du CHAPSA que ces services s'adressent d'alırd, tout en menant une enquête de proximité auprès des associations caritatives et d'autres SDF. Lorsque cette identité est établie, elle doit se charger de retrouver la famille pour l'avertir et qu'elle reconnaisse le corps. Dans ce dessein, ses moyens informatiques ne cépassant pas la grande couronne de Paris, et de nombreux SDF étant issus de province, l'enquête est souvent difficile : l'impossibilité du pouvoir s'appuyer sur des connexions éventuelles avec les recherches dans l'intérêt des familles que ces dernières auraient pu lancer, l'insuffisance des

9. Quelques thèses de médecine (en province) ont traité de cela, mais rien n'a été entrepris sur Paris Suite à ma demar de, le Dr J. Hassin, médecin au centre de soins du CHAPSA de Nanterre, a accepte de s'impliqur dans cette démarche. Depuis quatre années, nous avons dépouillé de multiples dossiers, pa fois difficiles à récupérer dans l'institution hospitalière. $\mathrm{Si}$ les données médicales sont importantes, les données sociologiques restent allusives. Le traitement et la :ynthese de ce travail est en cours. effectifs pouvant être affectés à cela, rendent la chose peu aisée. Il faut donc souligner que l'identification des SDF reste cruciale : les identités qu'ils donnent sont parfois fantaisistes ou se réduisent à des surnoms que leur vie à la rue leur a procurés. Le milieu associatif ne les désignant que par leur prénom ( «pour préserver leur pudeur » avoue un responsable), ce dernier sera d'un faible recours pour aider à cette identification sauf s'il accompagne régulièrement ces personnes. L'ensemble de ces raisons expliquent que nombre de SDF, morts dans la rue, sans papiers, soient finalement déclarés morts sous «X». À l'inverse, lorsque le corps trouvé peut être identifié grâce à des papiers, l'IML déclarera ce décès en faisant mention de la profession retrouvée dans ceux-ci alors que la personne ne l'exerce plus et vit à la rue depuis plusieurs années. Cette pratique, qui est au moins un déni de la réalité de la situation de la personne lors de son décès à la rue sinon, pénalement, une fausse déclaration, biaise encore un peu plus la possibilité d'analyser la mortalité des SDF sur le plan statistique.

- Lorsque la fanille $r_{i}$ 'a pas été retrouvée ou qu'elle refuse de prendre en charge les obsèques, ou bien encore lorsque le corps ne peut être identifié, le cadavre patiente en chambre froide à l'IML jusqu'à avoir sept partenaires pour justifier la mise en bière et le convoi, en camionnette banalisée, par la SAEM «Services funéraires de la ville de Paris », jusqu'au cimetière parisien de Thiais. Là, il est prucédé à l'inhumation de chacun dans un caveau à décomposition rapide ${ }^{10}$, sans rites particuliers. La démarche est la même pour la mort à l'hôpital. Cependant, si la demande est faite par des proches auprès de l'institution et sur la base d'un document attestant l'indigence du défunt, il est possible d'obtenir une levée du corps (avec porteurs en tenue et corbillard), un office religieux, et un convoi (qui peut accepter deux accompagnateurs jusqu'à Thiais), pour une inhumation plus ritualisée. Ces prestations sont alors facturées $609,80 €$, soit le double du coût de l'inhumation précédente, à la ville de Paris.

- Au bout de cinq années, les restes sont transférés et incinérés au crématorium du Père Lachaise, et les cendres disperséus dans le « Jardin du Souvenir ».

\section{PRÉCISIONS ET ÉVOLUTIONS}

Lors de cette enquête, ces caveaux numérotés étaient anonymes, et fort peu portaient la marque d'un souvenir quelconque. Ils témoigncut cependant, en

10. Il s'agit d'un caveau modulaire préfabriqué, conçu et réalisé par la société Augival. Son fonctionnement repose sur une circulation d'air dans le caveau, au moyen ae deux conduits en forme de siphons remplis de liquide bactériologique, obtenue de manière inerte par la différence de pression atmosphérique diurne et nocturne, accélérant la décomposition du corps. Un joint étanche est posé entre la dalle supérieure et le reste du coffret. Ces caveaux sont implantés dans les divisions n ${ }^{*} 48,49$ et 50 , dans un environnement paysager imposé par le cahier des charges, pour recycler les gaz de décomposition rejetés. 
succí dant à la fosse commune yui jusque-là accueillait les corps des plus démunis, d'une amélioration manifeste. Le corps repose dans un caveau individuel et n'est plus aligné, en terre, avec les autres, sur plusieurs niveaux. Il est plus facilement repérable, à partir du cahier d'enregistrement de la Conservation du cimetière et de fait, son exhumation éventuelle (pour identification) s'en trouve facilitée. Four les fossoyeurs comme pour les responsables des Cimetières parisiens, l'investissement concédé par la Ville de Paris " 1 est à la mesure des améliorations aussi bien techniques et éthiques que ces caveaux apportent.

Cependant, il faut apporter un bémol à cette efficacité technique appliquée à l'accélération du retraitement des corps : certains fossoyeurs témoignent qu'au bout de cinq ans, le résultat 7 'est pas toujours à la mesure des estimations. Plusieurs raisons techniques et culturelles peuvent expliquer cela. D'unc part, les différences entre la pression atmosphérique diurne et nocturne seraient moins importantes au nord de la Loire qu' au sud, atténuant ainsi l'optimisation du fonctionnement même du systènıe. D'autre part, les pratiques culturelles mortuaires en vigueur contrarient elles-mêmes cette logique : de fait, si habiller un cadavre pour l'inhumer ne facilite pas la circulation de l'air et sa décomposition rapide danśce type de caveau, sa disposition sous housse étanche, non biodégradable, par l'I IL interdit complètement celle-ci. «Il nous faudrait des cadavres nus pour arriver à un meilleur résultat » convient ụn fossoyeur

Interrogé réceınment sur ces dysfonctionnements éventuels et sur une enquête d'évaluation qu'ils auraient pu susciter, un responsable de ce secteur à la Mairie de Paris a semblé tomber des nues. Il ne serait pas sans intérêt qu' une Évaluation off. zielle irfirme ou confirme le constat des hommes de terrain qui se trouve d'ailleurs étayé, pour l' observateur anonyme, par l'adjonction discrète dans le caveau par un personnıl de SFVP, lors de l'inhumation, d'une petite boîte de substance non identifiable dont la fonction est sans doute de pallier cela.

Dans le champ symbolicue dont la pauvreté était à la mesure des défunts l'action menée par l'assuciat'on «Aux captifs la libération »12, permit d'obtenir de la part de la Mairie de Paris, des améliorations manifestes : depuis les plaques nominatives qui furent plać́s sur les caveaux dés l'été 2001, en passant par le dépôt d'une petite gerbc de fleurs sur chaque caveau lors de la fête de la Toussaint de cette même année, jusqu'à ia célébration laïque organisée et accueillie à l'Hôtel de Ville de Paris par M.B. Dılanoë, le 11 juin 2002 à la mémoire des morts de

11.Entre 1991 et 1994, la Ville de Paris a investi $3811225 €$ dans l'implantation de 1800 de ces caveaux. Depuis cette date, une seconde tranche de 228 caveaux a été réalisée en 2000 . et 330 nouveaux en 2001. La soc:été Augival est passée sous le contrôle de Vivendi.

12. Contacté par C. Rocra, chargée de mission sur ce sujet, en novembre 2000 , je lui avais donné un article (D. Terrolle, 1999) établissant la première version des résultats de ma recherche. C'est ce dernier qui servit de base à cette association pour susciter la campagne de presse qui alerta l'opinion publique en inai 2001 sur ce sujet. À propos de l'action de cette association, lire l'article de $C$. Rocca publié dars ce même numéro. la rue, le traitement funéraire de ces défunts s'est à l'évidence amélioré et une large prise de conscience, tant dans le public que dans l'institution municipale, s'est effectuée. Si l'anthropologue constate que ces personnes ne sont plus l'objet de la ségrégation qui les frappait même comme défunts, il remarque cependant que, avec la poursuite de cette ségrégation de leur vivant, l'absence de chiffres pertinents relatifs à leur décès persiste. Mais ceci d'une manière différente : au mutisme total précédent succède maintenant un «bruit » assourdissant, une profusion de chiffres qui, recoupés et analysés finement, restent contradictoires. Ainsi, sans doute pour faire la preuve que cette question n'est en rien redoutable, la « communication » produit une surenchère d'affirmations numériques, toutes plus affirmatives et diversement fondées les unes que les autres. Au silence de la dissimulation, devenu intenable en regard de l'action menée par cette association, succède le vertige des effets d'annonces qui est une autre manière de noyer le poisson. Le dossier de presse diffusé par la Mairie de Paris sur ce sujet lors de la dernière Toussaint est de ce point de vue édifiant: des nombres sont cités, des tábleaux chiffrés sont produits, et l'on retrouve même un item «SDF » dans ceux-ci... alors que la direction du SFVP déclare au chercheur, quelques mois après, qu'il est impossible de dégager significativement un item SDF parmi les défunts dont ils traitent les dossiers (lesquels servent de base de donnée exclusive à la Mairie de Paris). Avec cette surenchère numérique, un autre effet de cette campagne de presse s'est manifesté : ce sujet est devenu subitement "sensible » pour la Mairie de Paris, au point que l'accès du chercheur aux sources statistiques du SFVP (qui avant cela n'avait pas posé de problème) fait l'objet d'un entretien préalable à la demande du directeur de cette entreprise. La « transparence » affichée est donc loin de l'innocence première, et son aveu revendiqué officiellement avoue le « verrouillage» de cette question. Il ne manque plus que la nomination d'un comité de spécialistes pour parachever l'enterrement de ce nombre de morts derrière l'alibi de difficultés aussi techniques qu'éthiques.

On l'aura compris, si, dans le champ symbolique des choses ont changé, l'enjeu du nombre de ces décès reste le même. Pour quelle raison?

\section{LA MORT : UN ENJEU SOCIAL?}

S'interroger sur la dissimulation de ces chiffres implique de réfléchir, en parallèle, sur l'attitude très ambivalente de notre société vis-à-vis des autres décès : ainsi celle-ci adopte-t-elle en regard des diverses causes de mortalité qui frappent sa population des positions fort différentes. Dit autrement; toutes les causes de mortalité ne font pas l'objet, en France, de la même volonté statistique ni du même traitement médiatique.

De fait, l'enquête menée tant auprès de l'INSEE que de l'INED a mis en évidence, de la part des responsables cuntactés, le même constat déjà évoqué : se 
réfugiant derrière l'absence de «volonté politique » de faire avancer véritablement cctte question, ces spéciaiistes se renvoient la balle d'institution à institution.

D'autre part, pourquoi d'un point de vue médico-légal, aucune étude n'est parue sur cette question alors que l'Institut médico-légal occupe une place nodale dans la connaissance du nombre et de la cause de ces décès sur Paris?

Quant au traitement médiatique que notre société donne aux diverses causes de décès, on peut aussi remarquer qu'entre le nombre de décès par accidents de la circulation et ceux par suicide, son attitude varie considérablement. Si les fremiers sont largement publiés, les derniers sont occultés systématiquement. On ne peut alléguer pour expliquer cela une différence de pertinence statistique entre les deux : si les décès dus aux accidents de la circulation sont clairement enregistrés, ceux relevant du suicide, même s'ils restent pris dans des contextes qui influencent leur déclaration comme tel ${ }^{13}$ sont considérés comme pertinents.

Il faut donc chercher ailleurs les motivations de ces différences de traitement tant des groupes sociaux que des causes de décès, tant de la publicité qui s'attache à certains que de l'obstination à la dissimulation qui tente de masquer les autres.

C'est en cela qu'une anthropologie de la mort reste justement révélatrice du fonctionnement des vivants.

\section{QUEL NOMBRE DE MORTS?}

À la lumière des éléments frécédents, qu'est-ce qui serait à même de motiver de telles attitudes envers la mort des SDF ? S' agirait-il, en volume, de l'importance du nombre de ces décès ? Il est evident que la réponse primordiale réside là. Mais qu'apportera comme explication la révélation de ce nombre si l'on n'est pas en mesure de le rapporter à celui des SDF vivants ? de pouvoir tirer de ces rapports un pourcentage global et des pourcentages différenciés (par causes) de mortalité, et de les comparer avec ceux cui sont relatifs au reste de la population française?

Sans nul doute, l'obtention du nombre de décès de SDF, selon le sexe, l'âge, par mois et par année, sur Paris, permettrait de remettre en question bien des idées reçues (qu'entretiennent d'ailleurs de nombreuses campagnes caritatives) i commencer par celle, par exemple, de la forte mortalité hivernale qui frappe ces

13. Dans son rapport au Conseil Économique et Social sur «Le suicide» (rapport du 06/07/1993, J.O n० 15, 30/07/1993), M. Debout Évoque la pression des familles motivert du des raisons religieuses, sociales mais aussi économiques (relativement à une assurance vie) pour que le suicide ne soit pas déclaré comme tel. Pour ces raisons et d'autres liées à des dysfonctionnements inst itutionnels récurrents (comme le fréquent oubli des parquets, en cas d'au topsie, à renvoyer les bulletins de décès) il évalue alors à $20 \%$ la variation d'estimation du nombre des suicides er. France, chiffre qui, selon lui, ne remet pas en cause la pertinence des analyse: statistiques sur ce sujet. personnes alors que bien des éléments permettent de penser qu'au contraire, le pic de mortalité des SDF à Paris se situe à la période estivale, au mois d'août. Un autre intérêt serait d'anaiyser dans la durée, sur plusieurs années, la constance ou non de ces résultats. Enfin, l'étude des causes de la mortalité de ces personnes serait du plus vif intérêt pour éclairer tant la compréhension de leur mode de vie que celle de leur état sanitaire et épidémiologique, en regard de l'assistance qui leur est apportée.

Au-delà de cela, et pour faire un pas décisif de plus il faut que le nombre de ces décès puisse être rapporté à une évaluation pertinente dú nombre des SDF vivants, sur Paris. L'obtention de cette seconde référence n'est pas impossible à envisager même si la «liquidité " de cette population tant dans l'espace que dans le temps ne rend pas les choses aisées. Il serait alors possible de confirmer ou d'infirmer, pour Paris, les résultats produits par d'autres études menées à l'étranger sur cette question que, tant nos «spécialistes » des SDF que nos Observatoires de la pauvreté et que l'Institut médico-légal font semblant d'ignorer.

Ces travaux effectués à plus de vingt ans de distance et dans deux pays différents, en Suède et aux États-Unis, ne manquent pas pourtant d'intérêt dans la convergence de leurs résultats : en Suède, C.H. Alstrom, R. Lindelius et I. Salum, à partir de l'étude de six mille trente-deux dossiers de SDF, entre 1969 et 1971, dénombrent trois cent vingt-sept décès, soit une mortalité observée quatre fois plus élevé que la mortalité estimée. Ils mettent en évidence que la r.1ortalité par accident est douze fois plus élevée et celle, issue de maladies digestives ou respiratoires, sept fois plus élevée, que celle de la population de référence. Enfin, selon eux, la population SDF de moins de quarante ans a, globalement, un risque de décès neuf fois plus élevé que la population générale. Aux États-Unis, J. R. Hibbs, L. Benner, L. Klugman et d'autres, à partir d'une étude menée sur Philadelphie, soulignent en 1994 que les personnes itinérantes ont un taux de mortalité quatre fois plus élevé que dans le reste de la population. Autant dire que la situation de SDF ne rime pas avec l'importance de l'espérance de vie : tout le monde s'en doute mais personne ne le démontre. Pourquoi alors l'absence manifeste d'intérêt de ces institutions et de ces chercheurs, en France, pour travailler sur celle question?

\section{UNE HYPOTHÈSE TROUBLANTE}

L'ethnologue est bien obligé d'émettre, face à ce constat accablant, l'hypothèse selon laquelle tout se passe comme si, au-delà des apparences et des discours, la mortalité sans nul doute excessive des SDF représentait à la fois une chose impossible à avouer mais également une solution tacitement et collectivement acceptable. Cette attitude d'abjection sociale garantit en fait l'illusion que la solidarité que notre société nanifeste envers les SDF est pertinente. Le mutisme 
sur ces morts permet ainsi de justifier la pérennité de tout l'ensemble du dispositif en place : le traitement de l' «urgence sociale » tel qu'il est mis en place et défendu málgré ses résultats aberrants, mais aussi sa délégation par l'État au secteur associatif et caritatif qu'il finance directement en le subventionnant (sans obligation de résu!tats) et indirectement par le biais des déductions fiscales consenties aux dons humanitaires. Or, les aspects économiques de cette gestion de la pauvreté font apparaître une logique de marché, avec ses financements publics et privés, ses entreprises du social qui, au-delà des bénévoles magnifiques de dévouer.ent, ont une masse salariale croissante et des stratégies médiatiques et publicitaires qui témoignent de la concurrence existant pour se répartir les parts de ce marché. Pour que ce dernier prospère dans la «bonne conscience " générale, il est nécessaire que ces morts occultés, qui sont des témoins à charge de la faillite du dispositif et de sa logique, restent dans le silence institutionnel et social obstiné qui leur sert de lincelil. Ainsi on peut comprendre que si 94 à $95 \%$ des SDF ne se réinsèrent que par une mort rapide, l'efficacité économique de ce marché n'est pas à chercher dans ses résultats mais surtout dans l'imaginaire rassurant qu'il extretient relativement à la probabilité de ceux-ci.

Ainsi, se nouent dans cette hypothèse d'une part la dimension économique et sóciologique de cette question, et d'autre part la dimension du sacrés (laïc mais aussi religieux), dans le sens où, avec la mort, s'accomplit la logique sacrificielle ${ }^{14}$ dans laquelle s'inscrivent les SDF qui à ce terme seulement accèdent à une réinsertion. Lors de cette mort, ils renouent avec une identité, jusque-là déniée ou yerdue ; ils retrouvent une inscription administrative, un ancrage spatial et temporel officiel (même s'il ne dure que cinq années au cimetière de Thiais); ils sont resocialisés par des rites funéraires (certains réintègrent leur lignée dans le caveau familial) et par le devoir de mémoire que ceux-ci impliquent.

Cette sorte de bénéfice des victimes, rendues «consentantes» dans leur agonie par l'implacable logique du procédé 15 — «Il ne me reste plus qu'à mourir » concède avec juste sse un SDF épuisé et détruit par la vie à la rue - reste bien la moindre des choses que notre société puisse accorder à ces personnes en reconnaissance, sans doute inconsciente, de tout ce que leur mort nous permet de garantir : le détournement de la violence interne de la société (Girard R., 1972), notre « cohésion sociale », notre « lien social », notre « inclusion ». Sans parler de l'alibi aussi éthique qu'inattaquable qu'elles représentent pour justifier le marché de la pauvreté, le recyclage de nos stocks et de nos invendus alimentaires, et, finalement, notre «bonne conscience ».

14. Terrolle D., 1995, *'a liminarité des SDF. Rites de ségrégation et procédure sacrificielle 2 , Le Nouveau Mascaret, Bordc aux, 36, pp. 9-14.

15. Lempert B., 2000. Critique c'e la pensée sacrificielle, Paris, Seuil, 235 p.

\section{UN PACTE ABJECT..}

Cette abjection rencontrée en étudiant l'envers de la société, lors de cette recherche, n'est guère nouvelle pour les anthropologues dont le devoir est de construire leurs objets scientifiques au-delà des représentations séduisantes que toute société se complaît à donner d'elle-même.

En assistant à la célébration donnée pour les morts de la rue à l'Hôtel de Ville de Paris, le chercheur ne pouvait s'empêcher de penser à la pertinence de la remarque de r.. Lévi-Strauss (Lévi-Strauss C. 1955, 277) : « ... La représentation qu'une société se fait du rapport entre les vivants et les morts se réduit à un effort pour cacner, embellir ou justifier, sur le plan de la pensée religieuse, les relations réelles qui prévalent entre les vivants ». En célébrant ainsi leur méınoire, en évoquant leur fin, en chantant pour eux, qu'est-ce qui se ponctuait et se sanctifiait ainsi sinon, à travers ces victimes ainsi sacrifiées, le pacte nous liant au «Dieu obscur $\gg 10 . .$.

Daniel TERROLLE

Laboratoire d'Anthropologie Urbaine, CNRS

27 rue Paul Bert 94204 Ivrv sur Seine Cedex daniel.terrolle@wanadoofr

\section{BIBLIOGRAPHIE}

ALSTROM C.H., LINDELIUS R., SALUM I., 1975, Mortality Among Homeless Men, British Journal of Addiction, Alcohol and Other Drugs, $\mathrm{n}^{\mathrm{c}} 70, \mathrm{pp} .245-252$

BERGIER B., 2000, Les Affranchis. Paris, L'Harmattan, 207 p.

DAMON J., FIRDION J.M , 1996, Vivre dans la rue : la question SDF, in Paugam S. (sous la dir.), L'exclusion. L'étut des savoirs, Paris, La Découverte éd., 583 p.

DECLERCK P., 2001, Les Naufragés, Paris, Plon, 458 p.

DEBOUT, 1993, Le suicide, Rapport du Conseil Économique et Social, J.O. ${ }^{\circ} 15$. GIRARD R., 1972, La violence et le sacré, Paris, Grasset, 452 p.

HIBBS J.R., BENNER L., KLUGMAN L., SPENÇER R., MACCHIA I., MELLINGER

A.K., FIFE L., 1994, « Mortality in a Cotort of Homless Adults in Philadelphia », New England Journal of Medicine, $n^{\circ} 331$, pp. 304-309.

16. Zaloszyc A., 1994, Le Sacrifice au Dieu Obscur, Nice, Z'Editions, 95 p. 
LEMPERT B., 2000, Critique de la pensée sacrificielle, Paris, Seuil, 235 p.

LEVI-STRAUSS C., 1955, Trisi os tropiques, Paris, Plon, 490 p.

POUILLON J., 1977, «Plus c'est la même chose, plus ça change », Nouvelle Revue de Psychunalyse, 15.

TERROLLE D., Dir., 1993, Errances urbaines. Recherche en ethnologie urbaine. Rapport pour le Plan Urbain Construction et Architecture, $230 \mathrm{p}$.

TERROLLE D., 1995, « La liminarité des SDF. Rites de ségrégation et procédure sacrificielle ", Le Nouveau M Iascaret, $n^{\circ} 36$, pp. 9-14.

TERROLLE D., 1999, Privés de deuil, Le Nouveau Mascaret, n 55, pp. 26-32.

ZALOSZYC A., 1994, Le Sacriji :e au Dieu Obscur. Ténèbre et pureté dans la communauté. Nice Z'Editions, $95 \mathrm{p}$.

\section{RÉSUMÉ}

En mettant à jour l'escamotage systématique des données relatives à la mort des SDF à Paris, l'ethnologue questionne le mutisme délibéré de l'ensemble des institutions et des specialistes pourtant concernés par cette population. L'enquête de terrair. arrive à retracer le parcours de ces défunts, les difficultés rencontrés, les réti iences qu'elle suscite. Elle amène le chercheur à s'interroger sur l'enjeu rcdoutable que représentent ces morts: témoins à charge de la faillite d'un système qui les instrumentalise, mais aussi victimes sacrificielles nécessaires au maintien de la " cohésion sociale».

Mots-clés : SDF - Mort - Sacrifice.

\section{SUMMARY}

Datas of SDF (homeless) mortality seem to be systematically hidden in Paris The ethnologist tries to understand the reasons of this intentional muteness of institutions as specialists of this population. The fieldwork investigation recalls the course of these deceased, the problems and reserve that they generate. The researcher asks himself what kind of challenge those dangerous dead persons I epresent : witnesses of the failure of a Society that use them as objects, but also necessary victims of the maintenance of « social cohesion".

Key-words : Homeless - - Death - Sacrifice. 